\title{
Transcriptomic clues to understand the growth of Lactobacillus rhamnosus in cheese
}

\author{
Camilla Lazzi $^{1 *}$, Silvia Turroni ${ }^{2}$, Andrea Mancini ${ }^{1,3}$, Elisa Sgarbi $^{1}$, Erasmo Neviani $^{1}$, Patrizia Brigidi $^{2}$ and Monica Gatti ${ }^{1}$
}

\begin{abstract}
Background: Lactobacillus rhamnosus is a non-starter lactic acid bacterium that plays a significant role during cheese ripening, leading to the formation of flavor. In long-ripened cheeses it persists throughout the whole time of ripening due to its capacity to adapt to changing environmental conditions. The versatile adaptability of $L$. rhamnosus to different ecosystems has been associated with the capacity to use non-conventional energy sources, regulating different metabolic pathways. However, the molecular mechanisms allowing the growth of L. rhamnosus in the cheese dairy environment are still poorly understood. The aim of the present study was to identify genes potentially contributing to the growth ability of L. rhamnosus PR1019 in cheese-like medium (CB) using a transcriptomic approach, based on cDNA-amplified fragment length polymorphism (CDNA-AFLP) and quantitative real-time reverse transcription-PCR (qPCR).
\end{abstract}

Results: Using three primer combinations, a total of 89 and 98 transcript-derived fragments were obtained for L. rhamnosus PR1019 grown in commercial MRS medium and CB, respectively. The CDNA-AFLP results were validated on selected regulated genes by qPCR. In order to investigate the main adaptations to growth in a cheese-mimicking system, we focused on 20 transcripts over-expressed in CB with respect to MRS. It is worth noting the presence of transcripts involved in the degradation of pyruvate and ribose. Pyruvate is a intracellular metabolite that can be produced through different metabolic routes starting from the carbon sources present in cheese, and can be released in the cheese matrix with the starter lysis. Similarly the ribonucleosides released with starter lysis could deliver ribose that represents a fermentable carbohydrate in environments, such as cheese, where free carbohydrates are lacking. Both pyruvate degradation and ribose catabolism induce a metabolite flux toward acetate, coupled with ATP production via acetate kinase. Taking into account these considerations, we suggest that the energy produced through these pathways may concur to explain the great ability of L. rhamnosus PR1019 to grow on CB.

Conclusions: By a transcriptomic approach we identified a set of genes involved in alternative metabolic pathways in L. rhamnosus that could be responsible for L. rhamnosus growth in cheese during ripening.

Keywords: Lactobacillus rhamnosus, Cheese, cDNA-amplified fragment length polymorphism, Quantitative real-time reverse transcription-PCR

\section{Background}

Lactobacillus rhamnosus is a facultatively heterofermentative Lactic Acid Bacterium (LAB) frequently encountered in many dairy products, where it can be added as a probiotic microorganism or can be naturally present arising from raw milk. LAB may play different roles in cheese manufacture: some species participate in the

\footnotetext{
*Correspondence: camilla.lazzi@unipr.it

${ }^{1}$ Department of Food Science, Parma University, Parco Area delle Scienze 48/A, 43124 Parma, Italy

Full list of author information is available at the end of the article
}

fermentation process and contribute to acid production acting as starter LAB (SLAB), whereas others, called non-starter LAB (NSLAB), are mainly implicated in the maturation process. In particular, $L$. rhamnosus plays a significant role during ripening, leading to the formation of flavor $[1,2]$ and, for this reason, members of this species are generally recognized as NSLAB. It is noteworthy that NSLAB generally have a high tolerance to hostile environments, such as those with high salt concentration, low moisture, 4.9-5.3 $\mathrm{pH}$ values, low temperatures and deficiency of nutrients [3-5]. Moreover, several studies have reported that in long-ripened cheese varieties, 
NSLAB populations dominate during aging after SLAB decline due to autolysis [6,7]. Increasing by about four to five orders of magnitude within a few months, NSLAB can have a major impact in determining curd maturation and final characteristics of cheese [5]. In particular, L. rhamnosus has been shown to become dominant within NSLAB population in several cheeses, including Parmigiano Reggiano (PR) [8,9]. It persists throughout the whole time of PR cheese ripening (1 to 20 months) and this implies its capacity to adapt to changing environmental conditions [10]. Notably, different L. rhamnosus strains have been detected in relation to specific steps of cheese ripening, suggesting that these strains may have specific metabolic activities, which could account for the adaptation to the changing microenvironment of cheese ripening [11].

$\mathrm{Up}$ to date, the molecular mechanisms allowing the growth of L. rhamnosus in cheese are still poorly understood. NSLAB development during ripening can be attributed to their ability to use the major nutrient sources available in ripened cheese, that is lactose-free. The nutrient sources available include milk components modified by technological treatment (rennet addition and curd cooking) and starter LAB development, starter LAB metabolites and cell lysis products. Potential substrate for microbial growth are represented by small peptides or amino acids [4], citrate, lactate, and free fatty acids [12]. Additionally, sugars and phospholipids, nucleic acids and peptides can be released in the cheese matrix when SLAB autolysis starts to occur [13-15]. These compounds represent carbon sources that could yield intracellular pyruvate (i.e. through metabolism of citrate, lactate, amino acids, and nucleotides) or be converted into different metabolites.

To investigate the metabolic pathways occurring in L. rhamnosus during cheese ripening, Bove and colleagues [16] recently compared the proteomic profiles of $10 \mathrm{~L}$. rhamnosus strains grown in MRS and a cheese-like medium (Cheese Broth, CB). Differently from MRS, which is the standard laboratory medium for lactobacilli [17] and is considered to be a rich substrate with glucose as the primary carbon source for microbial growth, $\mathrm{CB}$ is an experimental medium formulated with 20 -monthripened PR cheese $[10,16,18]$, that tries to mimic the nutritional composition of PR cheese during ripening. PR cheese, and thus $\mathrm{CB}$, is considered to be a substrate poor in carbohydrates and characterized by the absence of milk sugar, lactose. During the curd acidification step of PR cheese production, the conversion of lactose into lactic acid is the main biochemical process that occurs. Lactose is completely depleted within 24 to $48 \mathrm{~h}$ [12]. The composition of $\mathrm{CB}$, prepared as the protocol of Neviani et al. [10], is the following ( $\mathrm{g} / \mathrm{l})$ : proteins, 80.92; lactose, 0.00; glucose, 0.00; galactose, 0.00 ; lactic acid, 3.82; $\mathrm{NaCl}, 3.4$; sodium citrate, 20.64 .
According to the findings of Bove et al. [16] compared to the cultivation in MRS, the differentially expressed proteins under cheese-like conditions were mainly linked to protein biosynthesis and catabolism, nucleotide and carbohydrate metabolisms, citrate metabolism, cell wall and exopolysaccharide biosynthesis, cell regulation, oxidation/reduction processes, and stress response [16]. Notably, L. rhamnosus produced lactic acid as the primary end product when growing in MRS, whereas in CB low levels of lactic acid together with high levels of acetic acid were detected for all strains. Despite this common trend, the authors also observed strain-specific physiological responses, suggesting a strain variability in the adaptation to changing environmental conditions in accordance with genetic polymorphism studies [11]. Among all strains, L. rhamnosus PR1019, isolated from PR 4-month-ripened cheese, has shown the greatest ability to growth in CB reaching after $48 \mathrm{~h}$ the highest cell numbers with one of the lowest levels of lactic acid and one of the highest levels of acetic acid.

For this reason, in the present work, we focused our attention only on this strain, with the aim to identify the genes that could concur to explain its growth ability in $\mathrm{CB}$ and its acid acetic production. The physiological adaptation of L. rhamnosus PR1019 in CB was evaluated using a transcriptomic approach, based on cDNA-amplified fragment length polymorphism (cDNA-AFLP) and quantitative real-time reverse transcription-PCR (qPCR). cDNA-AFLP is one of the most robust and sensitive transcriptomic technologies for genome-wide expression studies, with the main advantage of not requiring any prior knowledge of gene sequences while allowing the detection of lowly expressed genes through transcript amplification [19].

Using this approach, we identified a set of genes resulted over-expressed in CB compared to MRS, potentially involved in alternative metabolic pathways. Interesting genes were searched in other NSLAB and SLAB genomes with the aim to explore their diversity. Overall, the results described in this work highlight mechanisms of adaptation leading to the production of acetic acid coupled with ATP generation, that could support the L. rhamnosus growth in cheese during ripening.

\section{Methods}

\section{Bacterial growth conditions}

L. rhamnosus PR1019 was isolated from Parmigiano Reggiano (PR) at 4 months of ripening on cheese based medium [10] plate counts and identified by $16 \mathrm{~S} \mathrm{rDNA}$ gene sequencing [11] and species-specific PCR [20]. The strain was cultivated in MRS broth (Oxoid) or Cheese Broth $(\mathrm{CB})$ at $30^{\circ} \mathrm{C}$, under anaerobiosis, for 24 or $48 \mathrm{~h}$, respectively. $\mathrm{CB}$, a culture medium that mimics raw- 
milk long-ripened cheese, was prepared according to the modified protocol described by Bove et al. $[16,18]$.

\section{RNA extraction and CDNA synthesis}

The growth of L. rhamnosus PR1019 in MRS and CB broth was monitored by measuring optical density (OD) at $600 \mathrm{~nm}$. About $10^{9}$ cells at the top of logarithmic phase were harvested, and total RNA, stabilized with RNAprotect Bacteria Reagent (QIAGEN), was isolated using RNeasy Protect Bacteria Mini Kit (QIAGEN). Three independent biological experiment were made. RNA was quantified using a NanoDrop ND-1000 spectrophotometer (NanoDrop Technologies) and visualized by formaldehyde agarose gel electrophoresis according to standard procedures. All RNAs were of sufficient quantity $(>350 \mathrm{ng} / \mu \mathrm{l})$ and high quality (A260/A280 ratio 2.0 to 2.1). After a step of mRNA enrichment and polyadenylation of RNA transcripts, cDNA was synthesized by reverse transcription (RT) using a biotinylated oligo $(\mathrm{dT})$, following the protocol reported by Bove et al. [18]. For qPCR, single-stranded cDNA was prepared in a final volume of $20 \mu \mathrm{l}$ from total RNA using an oligo (dT) primer and SuperScript ${ }^{\mathrm{Tm}}$ II reverse transcriptase (Invitrogen), according to the manufacturer's instructions. Briefly, 12- $\mu \mathrm{l}$ reaction mixtures containing $500 \mathrm{ng}$ of oligo (dT) primer, $2 \mu \mathrm{g}$ total RNA and $10 \mathrm{nmol}$ dNTP mix in DEPC-treated $\mathrm{H}_{2} \mathrm{O}$ were heated to $65^{\circ} \mathrm{C}$ for $5 \mathrm{~min}$, added with $4 \mu \mathrm{l}$ of $5 \mathrm{X}$ First-Strand Buffer (Invitrogen) and $200 \mathrm{nmol}$ DTT, and then incubated at $42^{\circ} \mathrm{C}$ for $2 \mathrm{~min}$. RT reactions were started by the addition of $200 \mathrm{U}$ of enzyme, incubated at $42^{\circ} \mathrm{C}$ for $50 \mathrm{~min}$ and inactivated by heating at $70^{\circ} \mathrm{C}$ for $15 \mathrm{~min}$. RT step was carried out in duplicate.

\section{CDNA-AFLP}

cDNA-AFLP analysis was carried out as described by Bove et al. [18]. The protocol is based on the production of cDNA-AFLP fragments that are detected using infrared dye (IRD) detection technology and the Odyssey Infrared Imaging System. Briefly, after cDNA synthesis, a double digestion was carried out with EcoRI and MseI restriction enzymes and fragments were captured with the aid of streptavidin-coated magnetic beads. Digested cDNA fragments were subsequently ligated with adaptors to allow selective amplification with EcoRI primers labeled with an infrared dye (IRDye ${ }^{\mathrm{mm}} 700$ phosphoramidite), and unlabeled MseI-N (Eurofins MWG Operon). Three primer combinations were used to selectively amplify the expressed genes: DY-EcoRI-AC/ MseI-AT, DY-EcoRI-AT/MseI-AC and DY-EcoRI-AT/ MseI-AT [18]. Ligators and primers used are reported in Table 1. Separation of cDNA-AFLP fragments was carried out in a polyacrylamide gel and visualized by Odissey (LI-COR Biosciences) at $700 \mathrm{~nm}$.
Table 1 Primer and adaptor sequences

\begin{tabular}{lll}
\hline Primer/adaptor & Sequence (5'-3') & Application \\
\hline Adaptor EcoRI-f & CTCGTAGACTGCGTACC & Ligation \\
Adaptor ECoRI-r & AATTGGTACGCAGTCTAC & Ligation \\
Adaptor Msel-f & GACGATGAGTCCTGAG & Ligation \\
Adaptor Msel-r & TACTCAGGACTCAT & Ligation \\
EcoRI-0 & GACTGCGTACCAATTC & Non-selective PCR \\
Msel-0 & GATGAGTCCTGAGTAA & Non-selective PCR \\
5'DY-ECORI-AT & GACTGCGTACCAATTCAT & Selective PCR \\
5'DY-ECoRI-AC & GACTGCGTACCAATTCAC & Selective PCR \\
Msel-AT & GATGAGTCCTGAGTAAAT & Selective PCR \\
Msel-AC & GATGAGTCCTGAGTAAAC & Selective PCR \\
ECoRI-AC & GACTGCGTACCAATTCAC & Re-amplification PCR \\
ECoRI-AT & GACTGCGTACCAATTCAT & Re-amplification PCR \\
\hline
\end{tabular}

Primer sets were designed as reported by Bove et al. [18].

\section{CDNA-AFLP fragment isolation, re-amplification and} sequencing

Transcript-derived fragments (TDFs) of interest were cut from polyacrylamide gels as reported by Vuylsteke et al. [19], resuspended in $100 \mu \mathrm{l}$ of distilled water and subsequently re-amplified using the re-amplification and selective PCR primers EcoRI-AC/MseI-AT, EcoRI-AT/ MseI-AC and EcoRI-AT/MseI-AT (Table 1) according to the origin of cDNA-AFLP fragments. Amplification reactions were performed in a final volume of $50 \mu \mathrm{l} \mathrm{con-}$ taining $13 \mu \mathrm{l}$ of resuspended DNA fragment, $25 \mathrm{mM}$ $\mathrm{MgCl}_{2}, 10 \mathrm{X}$ PCR buffer, $2 \mu \mathrm{M}$ EcoRI-N primer, $2 \mu \mathrm{M}$ MseI-N primer, $5 \mathrm{mM}$ dNTPs, $0.5 \mu \mathrm{l}$ of AmpliTaq 360 DNA polymerase $(5 \mathrm{U} / \mu \mathrm{l})$ and $2 \mu \mathrm{l}$ of $360 \mathrm{GC}$ enhancer (Applied Biosystems-Life Technologies). PCR consisted of: i) $30 \mathrm{~s}$ of denaturation step at $94^{\circ} \mathrm{C}, 30 \mathrm{~s}$ of annealing step at $65^{\circ} \mathrm{C}$ (reduced of $0.7^{\circ} \mathrm{C}$ at each cycle) and $1 \mathrm{~min}$ of extension step at $72^{\circ} \mathrm{C}$ for 13 total cycles; ii) $10 \mathrm{~s}$ of denaturation step at $94^{\circ} \mathrm{C}, 30 \mathrm{~s}$ of annealing step at $56^{\circ} \mathrm{C}$ and $1 \mathrm{~min}$ of extension step at $72^{\circ} \mathrm{C}$ (extended for $1 \mathrm{~min}$ at each cycle) for 35 total cycles. These cycles were preceded by a common denaturation step of $2 \mathrm{~min}$ at $94^{\circ} \mathrm{C}$ and followed by a final 10 -min extension at $72^{\circ} \mathrm{C}$, and were carried out in a Mastercycler ep gradient $\mathrm{S}$ thermal cycler (Eppendorf). Amplified products were checked on a $1 \%$ agarose gel with a 100-bp marker (Invitrogen) and subsequently purified using the Wizard ${ }^{\circ}$ SV Gel and PCR Clean-Up System according to the manufacturer's instructions (Promega Corporation). Amplified fragments were then cloned in E.coli using the pGEM-T Easy Vector System kit (Promega Corporation), and plasmids from selected clones were purified using PureYield MiniPrep System kit (Promega Corporation) referring to the producer's manual. Cloned fragments were finally sequenced by Eurofins MWG Operon using primers M13 
and sequences were analysed by BLAST alignment [21]. TDF sequences were deposited in the DDBJ database under the accession numbers AB896768 to AB896786.

\section{qPCR and data processing}

qPCR was carried out using the LightCycler SYBR Green system (Roche) as previously described [22]. Briefly, $1 \mu \mathrm{l}$ of cDNA template was used in each reaction along with $4 \mu \mathrm{l}$ of SYBR Green PCR master mix (Roche) and $10 \mathrm{pmol}$ of the appropriate gene-specific primers in a final volume of $20 \mu \mathrm{l}$. The following cycle profile was used: $10 \mathrm{~min}$ at $95^{\circ} \mathrm{C}, 40$ repeats of $15 \mathrm{~s}$ at $95^{\circ} \mathrm{C}, 25 \mathrm{~s}$ at $58^{\circ} \mathrm{C}$ for $\operatorname{sp} x B$, ulaE and $16 S \mathrm{rDNA}$ genes or $55^{\circ} \mathrm{C}$ for $x f p, 72^{\circ} \mathrm{C}$ for $20 \mathrm{~s}(30 \mathrm{~s}$ for $16 S \mathrm{rDNA}$ ) and an additional 5-s incubation step at $81^{\circ} \mathrm{C}$ for fluorescence acquisition. Oligonucleotide sequence information and detailed primer-specific conditions are given in Table 2. Two technical replicates were done for each combination of cDNA and primer pair. To assess background and residual DNA contamination, a no-template control (NTC) and a no-reverse transcription control (NoRT) were performed for each target. DNA contamination was considered to be negligible when the difference in $\mathrm{C}_{\mathrm{q}}$ (quantification cycle) between the sample and the respective NoRT was above 5 cycles. Product detection and PCR specificity were checked post-amplification by examining the dissociation curves. PCR amplicons were resolved by $2 \%$ agarose gel electrophoresis to verify the expected size. To evaluate repeatability and reproducibility of the qPCR assay, intra- and inter-assay coefficients of variation $(\mathrm{CV})$ were assessed. The intra-assay $\mathrm{CV}$ was from 0.7 to $7.6 \%$ whereas the inter-assay CV ranged from 8.3 to $18.8 \%$. Amplification efficiency was calculated from the slope of standard curves generated with two-fold serial dilutions of the same cDNA sample, as $\mathrm{E}=10^{(-1 / \text { slope })}$. Relative expression of target genes was determined using the $\Delta \Delta C_{\mathrm{T}}$ method after Pfaffl correction [23]. 16S rDNA was used as a reference gene.

\section{In silico analysis}

TDF sequences were annotated using BLAST search. Pathway assignment was performed according to COG (Cluster of Orthologous Groups) [25] functional categories and KEGG (Kyoto Encyclopedia of Genes and Genome) [26] pathway database. Gene synteny across NSLAB and SLAB genomes was explored through the web server SyntTax [27]. Genome mining for promoter and terminator elements was performed using PePPER toolbox [28]. Translated protein sequences were subjected to Pfam motif analysis [29]. Protein alignments were performed using ClustalW2 [30] and used for phylogenetic tree construction at the Interactive Tree of Life [31]. Multisequence amino acid alignments were represented using CLC-Bio sequence viewer [32].

\section{Results and discussion cDNA-AFLP analysis}

In this study, the cDNA-AFLP technique [18] was applied to profile the transcriptome of a $L$. rhamnosus strain grown in conditions mimicking cheese ripening. Despite it is not widely used in bacteria, cDNA-AFLP can be considered an ideal system for genome-wide expression analysis, mainly for the detection of lowly expressed genes.

Three primer combinations were used to selectively amplify the genes expressed by L. rhamnosus PR1019 in $\mathrm{CB}$ and MRS, allowing to generate different cDNA-AFLP profiles with a fragment size ranging from 50 to $500 \mathrm{bp}$ (Figure 1). A total of 89 and 98 TDFs were detected in MRS and $\mathrm{CB}$, respectively. In order to investigate the main adaptations of L. rhamnosus to the PR cheese environment, we focused on TDFs over-expressed in CB.

\section{Identification of TDFs over-expressed in CB}

Twenty TDFs strongly over-expressed by L. rhamnosus in $\mathrm{CB}$ compared to MRS were extracted from gel and used as templates for re-amplification by PCR.

Table 2 qPCR settings and relative transcript abundance

\begin{tabular}{|c|c|c|c|c|}
\hline Target gene & Primer sequence $\left(5^{\prime}-3^{\prime}\right)^{a}$ & Product size (bp) & PCR efficiency & Expression ratio $^{\mathrm{b}}$ \\
\hline \multirow[t]{2}{*}{$s p \times B$} & fwd: TACCGGAAACTGCTTGGTATC & 155 & 1.93 & 8.97 \\
\hline & rev: CTGGAAAACCGCATCTTTGT & & & \\
\hline \multirow[t]{2}{*}{ ulaE } & fwd: CACTAGCCAAATCAATCGCC & 90 & 2.05 & 5.78 \\
\hline & rev: GCCATCGTCGGTTTCCATTA & & & \\
\hline \multirow[t]{2}{*}{$x f p$} & fwd: CGTGAAGAAGGCGATATC & 215 & 2.01 & 5.98 \\
\hline & rev: TTCCAAGTCCACTCCTGA & & & \\
\hline \multirow[t]{2}{*}{$165 \mathrm{rDNA}$} & fwd: GCYTAACACATGCAAGTCGA & 500 & 1.85 & / \\
\hline & rev: GTATTACCGCGGCTGCTGG & & & \\
\hline
\end{tabular}

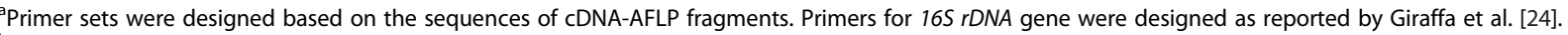

${ }^{\mathrm{b}}$ Target gene expression was calculated relative to $16 \mathrm{~S} r D N A$ as a reference gene using the efficiency-corrected $\Delta \Delta C_{\mathrm{T}}$ method [23]. The relative expression ratios in CB compared to MRS are shown. 


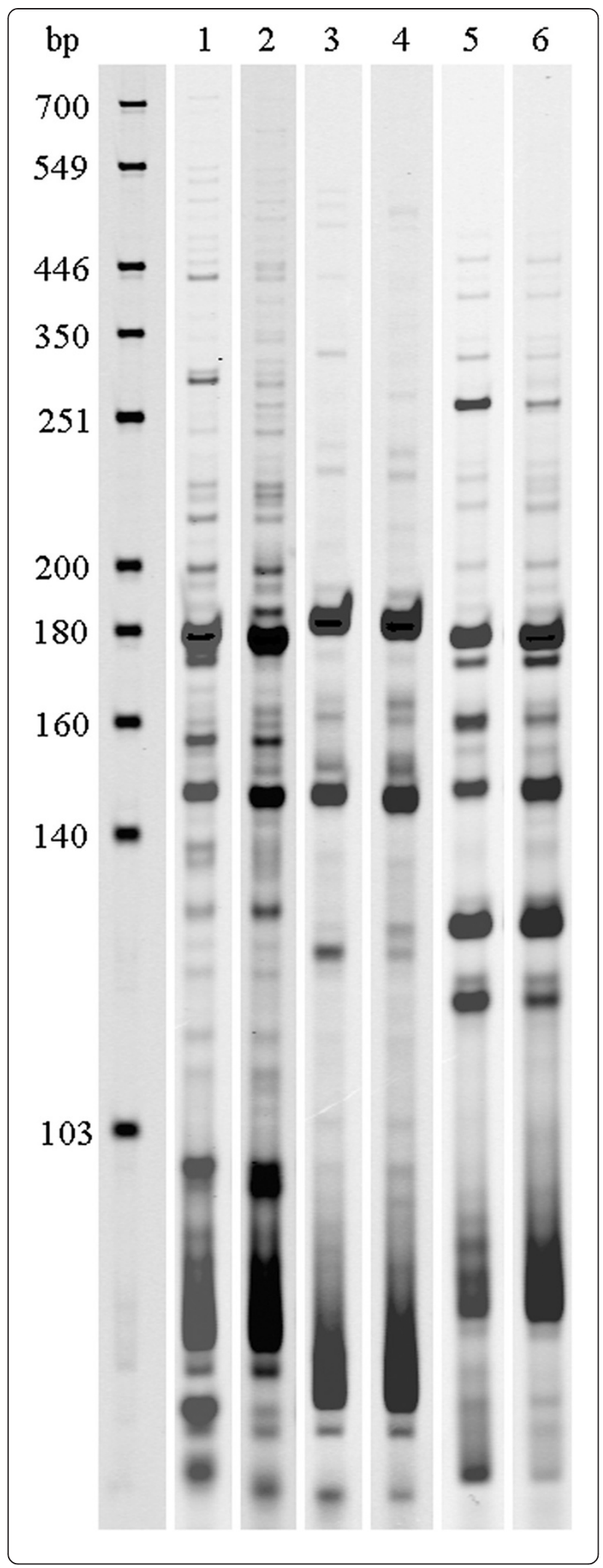

Figure 1 CDNA-AFLP fingerprint of $L$. rhamnosus PR1019 grown in MRS and $C B$, obtained with three different primer combinations. M, 50-700 bp IRDye700 Sizing Standard; lanes 1, 3 and 5, cDNA-AFLP fingerprinting of $L$. rhamnosus cultured in MRS using EcoRI-AC/Msel-AT, EcoRI-AT/Msel-AC and EcoRI-AT/Msel-AT primer combination, respectively; lanes 2, 4 and 6, CDNA-AFLP fingerprinting of L. rhamnosus cultured in CB using EcoRI-AC/Msel-AT, EcoRI-AT/Msel-AC and EcoRI-AT/ Msel-AT primer combination, respectively.

Direct sequencing of the PCR products failed probably because the sequences were contaminated with comigrating bands or were too small (data not shown). Products from bands were then cloned and PCR amplicons from 10 individual colonies were sequenced. Reliable (> $100 \mathrm{bp}$ ) sequences were obtained for 19 of the 20 TDFs. Each sequence was identified by similarity search using the BLAST program against the GenBank nonredundant (nr) public sequence database.

As shown in Table 3, 8 transcripts (approximately 42\% of selected sequences) showed a significant similarity to sequences with known function, 5 transcripts $(26.5 \%$ of selected sequences) were closely related to $L$. casei plasmid sequences, 4 transcripts ( $21 \%$ of selected sequences) were annotated as hypothetical protein-coding sequences, and 2 transcripts (10.5\% of selected sequences) were identified as 5S rRNA.

It is known that plasmids often carry genes that might be essential for survival under harsh conditions, encoding important traits, such as enzymes involved in secondary metabolic pathways [33]. Plasmids are known to be a source of LAB genetic and phenotypic diversity which occasionally confers adaptive advantages to host strains [34]. However, further studies are clearly needed to better explore the role of plasmid sequences in the L. rhamnosus adaptation to the cheese ripening environment. To validate the cDNA-AFLP expression profiles, 3 genes, encoding pyruvate oxidase $(\operatorname{sp} x B)$, L-xylulose 5-phosphate 3-epimerase (ulaE), and xylulose-5-phosphate phosphoketolase $(x f p)$ were selected for qPCR. The relative mRNA abundances were normalized by that of the commonly used reference gene $16 S \mathrm{rDNA}$, and expressed as a ratio of $\mathrm{CB}$ to MRS levels. Amplification efficiency for all assays ranged between 85 and $105 \%$. Confirming the reliability of cDNA-AFLP results, all transcripts were more abundant in $\mathrm{CB}$, with expression ratios over 5-fold (Table 2).

To investigate a possible role for these genes in allowing L. rhamnosus growth in cheese during ripening, in silico analyses were carried out.

\section{SpxB}

In silico analysis of TDF no. 93 (305 bp), encoding 101 amino acid residues, revealed the highest identity in amino acid sequence (93\%) with a pyruvate oxidase 
Table 3 Transcript-derived fragments (TDFs) from L. rhamnosus PR1019 over-expressed in CB compared to MRS

\begin{tabular}{|c|c|c|c|c|c|c|c|c|}
\hline \multirow{2}{*}{$\begin{array}{l}\text { TDF } \\
\text { no }\end{array}$} & \multirow{2}{*}{$\begin{array}{l}\text { Primer } \\
\text { combination }\end{array}$} & \multirow{2}{*}{$\begin{array}{l}\text { Length } \\
\text { (bp) }\end{array}$} & \multirow[t]{2}{*}{ Biological function $^{a}$} & \multirow[t]{2}{*}{ Organism annotation $^{\mathbf{b}}$} & \multirow{2}{*}{$\begin{array}{l}\text { Max identity - } \\
\text { E-value }^{c}\end{array}$} & \multirow[t]{2}{*}{ Accession no. } & \multicolumn{2}{|l|}{ Pathway assignment $^{\mathrm{d}}$} \\
\hline & & & & & & & $\operatorname{COG}^{\mathrm{e}}$ & KEGG \\
\hline 37 & AC/AT & 396 & Guanylate kinase (EC 2.7.4.8) & L. rhamnosus GG & $98 \%-1 e-84$ & YP_003171760.1 & COG0194 [F] & $\begin{array}{l}\text { ko00230: Purine } \\
\text { metabolism }\end{array}$ \\
\hline 40 & AC/AT & 302 & $\begin{array}{l}\text { Putative phosphoketolase } \\
\text { (EC 4.1.2.9) }\end{array}$ & L. rhamnosus GG & $99 \%-3 e-57$ & YP_005864692.1 & COG3957 [G] & $\begin{array}{l}\text { ko00030: Pentose } \\
\text { phosphate } \\
\text { pathway }\end{array}$ \\
\hline 48 & AC/AT & 199 & Monooxygenase & L. rhamnosus LC 705 & $95 \%-6 e-17$ & YP_003174467.1 & $\begin{array}{l}\text { COG2329: Conserved protein } \\
\text { involved in polyketide } \\
\text { biosynthesis related to } \\
\text { monooxygenase }[R]\end{array}$ & - \\
\hline 54 & AC/AT & 137 & Hypothetical protein & L. rhamnosus & $77 \%-2 e-07$ & WP_005689523.1 & - & - \\
\hline 72 & AC/AT & 340 & $\begin{array}{l}\text { Lipoteichoic acid synthase LtaS } \\
\text { Type Ila (EC 3.1.6) }\end{array}$ & L. rhamnosus LC 705 & $100 \%-5 e-43$ & YP_003173514.1 & $\begin{array}{l}\text { COG1368: Phosphoglycerol } \\
\text { transferase and related proteins, } \\
\text { alkaline phosphatase } \\
\text { superfamily }[\mathrm{M}]\end{array}$ & - \\
\hline 76 & AC/AT & 433 & Conserved hypothetical protein & L. rhamnosus LC 705 & $85 \%-9 e-27$ & YP_003174890.1 & - & - \\
\hline 86 & AC/AT & 109 & $\begin{array}{l}\text { L-xylulose 5-phosphate 3-epimerase } \\
\text { (EC 5.1.3.22) }\end{array}$ & L. rhamnosus GG & $94 \%-9 e-13$ & YP_003172471.1 & $\operatorname{coG} 3623[\mathrm{G}]$ & $\begin{array}{l}\text { ko00040: Pentose and } \\
\text { glucuronate } \\
\text { interconversions }\end{array}$ \\
\hline 93 & AC/AT & 305 & Pyruvate oxidase (EC 1.2.3.3) & L. rhamnosus GG & $93 \%-5 e-40$ & YP_003171582.1 & $\begin{array}{l}\text { COG3961: Pyruvate decarboxylase } \\
\text { and related thiamine } \\
\text { pyrophosphate-requiring } \\
\text { enzymes [G] }\end{array}$ & $\begin{array}{l}\text { ko00620: Pyruvate } \\
\text { metabolism }\end{array}$ \\
\hline 95 & AC/AT & 229 & Plasmid pNCD0151 & L. casei & $98 \%-4 e-68$ & Z50861.1 & - & - \\
\hline 97 & AC/AT & 227 & Plasmid pNCD0151 & L. casei & $96 \%-3 e-64$ & Z50861.1 & - & - \\
\hline 106 & AC/AT & 170 & Plasmid pNCD0151 & L. casei & $97 \%-9 e-48$ & Z50861.1 & - & - \\
\hline 120 & AC/AT & 107 & Hypothetical protein & L. casei & $96 \%-4 e-10$ & WP_003574536.1 & - & - \\
\hline 121 & AC/AT & 105 & $\begin{array}{l}\text { Imidazoleglycerol-phosphate } \\
\text { dehydratase (EC 4.2.1.19) }\end{array}$ & L. rhamnosus LC 705 & $92 \%-5 e-08$ & YP_003174148.1 & COG0131 [E] & $\begin{array}{l}\text { ko00340: Hystidine } \\
\text { metabolism }\end{array}$ \\
\hline 122 & AC/AT & 102 & Plasmid pNCD0151 & L. casei & $96 \%-5 e-23$ & Z50861.1 & - & - \\
\hline 162 & AT/AC & 350 & $\begin{array}{l}\text { Calcineurin-like phosphoesterase } \\
\text { family protein }\end{array}$ & L. rhamnosus ATCC 8530 & $97 \%-3 e-70$ & YP_005872999.1 & $\begin{array}{l}\text { COG0737: 5'-nucleotidase/2', } \\
\text { 3'-cyclic phosphodiesterase } \\
\text { and related esterases [F] }\end{array}$ & - \\
\hline 168 & AT/AC & 238 & Plasmid pNCD0151 & L. casei & $98 \%-1 e-68$ & Z50861.1 & - & _- \\
\hline
\end{tabular}


Table 3 Transcript-derived fragments (TDFs) from L. rhamnosus PR1019 over-expressed in CB compared to MRS (Continued)

\begin{tabular}{lllllll}
\hline 170 & AT/AC & 222 & Hypothetical protein & L. rhamnosus GG & $98 \%-5 \mathrm{e}-34$ & YP_003171844.1 \\
211 & AT/AT & 240 & 5S ribosomal RNA & L. rhamnosus GG & $98 \%-2 \mathrm{e}-11$ & NR_103302.1 \\
212 & AT/AT & 234 & 5S ribosomal RNA & L. rhamnosus GG & $98 \%-4 \mathrm{e}-09$ & NR_103302.1 \\
\hline
\end{tabular}

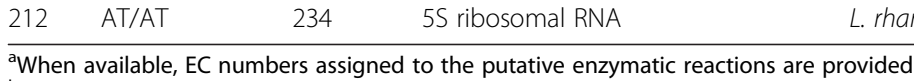

${ }^{\text {b} T h e ~ c o l u m n ~ i n d i c a t e s ~ t h e ~ m i c r o o r g a n i s m ~ o f ~ t h e ~ b e s t ~ h i t ~ f r o m ~ B L A S T X ~ s e a r c h ~}$

${ }^{c}$ Max identity and E-value from the best hit of BLASTX search are provided.

"Max identity and E-value from the best hit of BLASTX search are provided.

${ }^{e} E$, Amino acid transport and metabolism; $F$, Nucleotide transport and metabolism; $G$, Carbohydrate transport and metabolism; M, Cell wall/membrane/envelope biogenesis; R, General function prediction only. 
(SpxB) from L. rhamnosus GG (Table 3). Lower levels of identity were observed for SpxB of other members of L. casei group (L. casei, 79\%; L. paracasei subsp. paracasei, 79\%; L. zeae, 75\%). BLASTX search also returned a number of pyruvate oxidases of other NSLAB, such as L. curvatus (55\%), L. buchneri (46\%), L. brevis (46\%), L. plantarum (41\%) and L. pentosus (41\%), as well as of non-Lactobacillus bacteria.

$\mathrm{SpxB}$ is an enzyme involved in the pyruvate metabolism pathway. $\mathrm{LAB}$ can metabolize pyruvate into lactate by lactate dehydrogenase (LDH) or into acetate via pyruvate formate lyase (PFL), phosphotransacetylase (PTA) and acetate kinase (ACK), or via pyruvate oxidase (POX) pathway [35]. In the latter, pyruvate is oxidized with the production of hydrogen peroxide and acetyl phosphate, followed by acetate production and ATP generation via ACK (Figure 2).

To our knowledge, this is the first evidence of activation of the POX pathway in L. rhamnosus. On the contrary, POX activity has been extensively described to date in L. plantarum and involved with acetate production in its survival during the stationary phase of aerobic growth [35-39]. In particular, accumulation of acetate instead of lactate is thought to play a role in ensuring the $\mathrm{pH}$ homeostasis with an overall beneficial effect for the cell $[37,40]$. The additional ATP generated via ACK has been shown to enhance the biomass production [41]. Interestingly, Lorquet et al. [37] showed that in the late stationary phase, when the production of acetate stopped, an OD decrease resulting from lytic processes occurred. The hypothesis is that in the absence of ATP production, protons can no longer be extruded by ATPases with a consequent dissipation of the proton motive force, which has been shown to be one of the mechanisms triggering autolysis of gram-positive bacteria.

Interestingly, high levels of acetic acid and low levels of lactic acid have been recently observed in L. rhamnosus strains grown in $\mathrm{CB}$ under the same conditions of our study [16,42] Furthermore, by a proteomic approach, Bove et al. [16] showed an increase in expression of PTA and ACK, which are involved in the synthesis of acetic acid in a branch of the pyruvate metabolism other than POX pathway (Figure 2), during L. rhamnosus growth in CB compared to MRS. Highlighting a possible alternative route of degradation of pyruvate to acetate (the POX pathway; Figure 2), our transcriptomic results seem to complement data from proteomics, strengthening the hypothesis that L. rhamnosus can utilize pyruvate as a growth substrate during cheese ripening.

Pyruvate is an intracellular metabolite that could be produced through different metabolic routes using the carbon sources present in cheese (i.e. through metabolism of citrate, lactate, amino acids, and nucleotides). Moreover, pyruvate can be released in the cheese matrix with starter lysis. Liu et al. [43] showed that the activity of POX in L. plantarum could be related to the catabolism of L-serine. According to the authors, L-serine is deaminated via a serine dehydratase into pyruvate, which is subsequently converted into acetate by the POX enzyme [43]. Pyruvate conversion by POX has been recently supposed also in $L$. casei [44]. Looking at the closely related taxonomic groups of these heterofermentative lactobacilli and considering that they share similar ecological niches, including ripened cheese, it appears interesting to further investigate in the future the role of pyruvate metabolism during cheese ripening.

Notably, 3 genes encoding putative pyruvate oxidases are harbored in the completely sequenced genomes of L. rhamnosus GG and L. casei ATCC 334, whereas 4 and 5 pox genes were retrieved in the genome sequences of L. buchneri CD034 and L. plantarum WCFS1, respectively. Goffin et al. [36] reported that among the predicted pox genes encoded in the L. plantarum lp80 genome, only poxB and pox $F$ appeared to be involved in the generation of acetate from lactate during the stationary phase of aerobic growth. Interestingly, $p \circ x B$ and

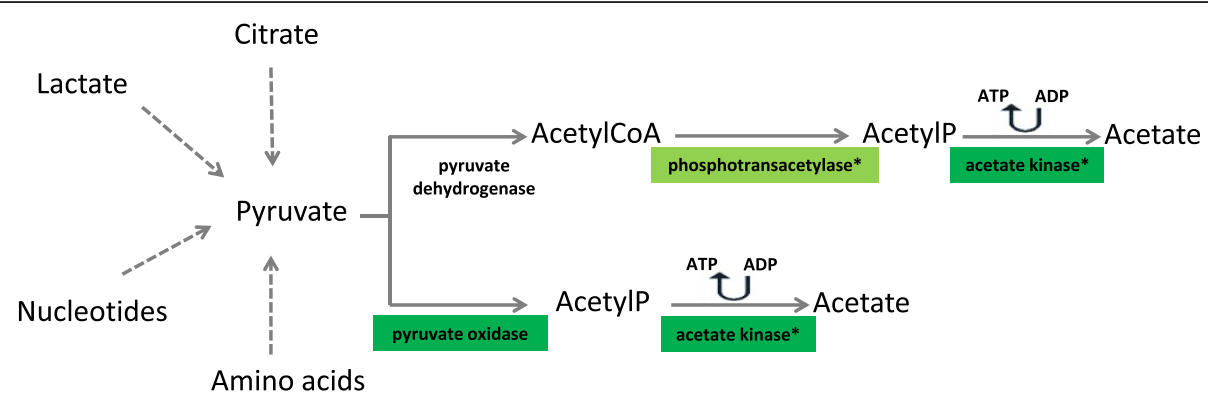

Figure 2 Conversion of pyruvate into acetate. Enzymes showing differences in protein $\left(^{*}\right)$ or transcript abundance for L. rhamnosus PR1019 grown in CB compared to MRS are highlighted. Dark green, expression ratio CB versus MRS 5 to 10 ; light green, expression ratio CB versus MRS < 5 . Transcript data are from the present study. Protein data are from Bove et al. [16]. 
pox $F$ genes shared 63 and $61 \%$ amino acid similarity with TDF 93, respectively. To date, only one gene potentially encoding for pyruvate oxidase has been located in the complete genome sequences of the SLAB L. helveticus R0052 and L. delbrueckii subsp. bulgaricus ATCC 11842. The pyruvate oxidase gene of $L$. rhamnosus GG with the highest homology to TDF 93 is flanked by genes whose order and transcriptional orientation are partially shared with $L$. casei ATCC 334 but not with L. buchneri CD034, L. plantarum WCFS1, L. helveticus R0052, L. delbrueckii subsp. bulgaricus ATCC 11842 and L. brevis ATCC 367 (Figure 3A). In particular, sp xB locus in $L$. rhamnosus and $L$. casei genomes is preceded by three genes encoding putative hydroxymethylglutarylCoA synthase, hydroxymethylglutaryl-CoA reductase and acetyl-CoA acetyltransferase. These enzymes are known to be involved in the mevalonate pathway, routing acetyl-CoA towards isoprenoid biosynthesis. However, whether these proteins are actually expressed in L. rhamnosus and play a role in deviating the flow of acetyl-CoA from the acetate production via PTA and ACK during cheese ripening still remain to be determined. According to PePPER, $s p x B$ gene from L. rhamnosus GG was predicted to be monocistronically transcribed. Phylogenetic tree showed a clear segregation of putative pyruvate oxidases from $L$. casei group (Figure 4A). As expected, a subgroup was represented by POX proteins from the SLAB L. helveticus, L. delbrueckii subsp. bulgaricus and L. delbrueckii subsp. lactis. L. plantarum and L. pentosus homologues clustered together and close to L. buchneri. Multiple sequence alignment of TDF 93 and pyruvate oxidase protein sequences from several NSLAB and SLAB is shown in Additional file 1: Figure S1A.

\section{UlaE}

BLASTX analysis of TDF no. 86 (109 bp), putatively encoding 36 amino acid residues, showed the maximum identity (94\%) to a protein annotated as L-xylulose 5phosphate 3-epimerase (ulaE) from L. rhamnosus GG (Table 3). Eighty-four percent of identity was exhibited to the same putative protein from other $L$. casei group members ( $L$. casei and $L$. paracasei subsp. paracasei). Homologues were also found in NSLAB known to play a role in flavor generation and other ripening processes: L. suebicus (74\%), L. coryniformis (72\%) and Carnobacterium maltaromaticum (69\%).

$\mathrm{UlaE}$ is an epimerase involved with other enzymes (UlaD and UlaF) in the production of D-xylulose 5phosphate $[45,46]$, an intermediate in the pentose phosphate pathway.

According to SyntTax, regions up and downstream of ulaE gene from L. rhamnosus GG shared a conserved gene order with $L$. casei ATCC 334, whereas no synteny was found in L. buchneri CD034, L. plantarum WCFS1, L. helveticus R0052, L. delbrueckii subsp. bulgaricus ATCC 11842 and L. brevis ATCC 367 genomes (Figure 3B). According to PePPER analysis of L. rhamnosus GG genome, a potential terminator stem-loop structure was identified $82 \mathrm{bp}$ downstream from the $a r a D$ gene stop codon. No putative promoters were predicted up to $5000 \mathrm{bp}$ upstream of ulaE gene. Interestingly, the upstream LGG_02727 gene was annotated as a transcriptional regulator, belonging to DeoR family. Phylogenetic analysis of L-xylulose 5phosphate 3-epimerase homologues revealed that ulaE predicted protein from L. rhamnosus clustered close to the putative enzymes from other $L$. casei group members and L. coryniformis (Figure 4B). Multiple sequence alignment of TDF 86 and homologs from several NSLAB is shown in Additional file 1: Figure S1B.

\section{Xfp}

TDF no. 40 (302 bp) displayed the highest identity (99\%) in amino acid sequence with a putative phosphoketolase (xfp) from L. rhamnosus GG (Table 3). Percentages of identity $>95 \%$ were found with other $L$. casei group members (L. zeae, 98\%; L. paracasei subsp. paracasei, 96\%; L. casei, 96\%). BLASTX search also revealed a significant match to a predicted xylulose-5-phosphate phosphoketolase from L. coryniformis (identity 75\%). Interestingly, lower levels of identity were obtained with SLAB, such as L. delbrueckii subsp. bulgaricus (56\%), L. delbrueckii subsp. lactis (56\%) and L. helveticus (55\%).

Facultatively heterofermentative LAB, like L. rhamnosus, degrade hexoses via the Embden-MeyerhoffParnas pathway and pentoses via the phosphoketolase pathway (PKP). Xylulose 5-phosphate phosphoketolase is the central enzyme of PKP. In the presence of inorganic phosphate this enzyme converts xylulose 5-phosphate into glyceraldehyde 3-phosphate and acetylphosphate (Figure 5) [47]. Recently, McLeod et al. [48] studied the transcriptome response of $L$. sakei during growth on ribose, demonstrating that the ribose uptake and catabolic machinery are highly regulated and closely linked with the catabolism of nucleotides. It is known that ribonucleosides are source of ribose as a fermentable carbohydrate in environments where free carbohydrates are lacking. For example, in the meat, a rich environment but carbohydrate-poor substrate for microorganisms, the ability of $L$. sakei to use nucleosides offers a competitive advantage [49]. Nucleosides represent a potential energy source also in the cheese environment, where microbial autolysis occurs, releasing ribose- and desoxyribosecontaining nucleic acids [14]. Notably, it has been observed that ribose released after lysis of SLAB decreased steadily in parallel with the growth of facultatively heterofermentative lactobacilli, strongly suggesting that these bacteria used ribose as a growth substrate [14]. 


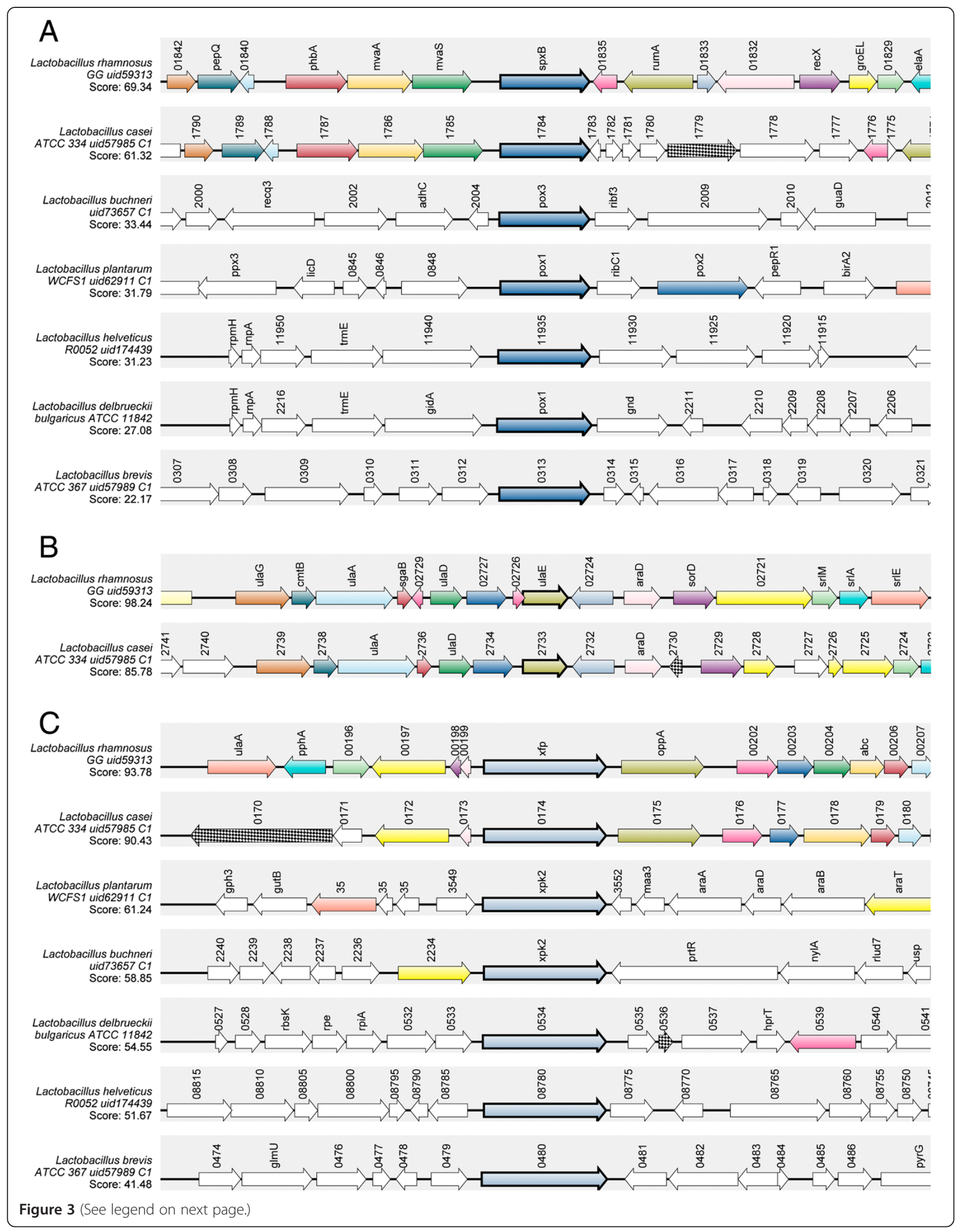


(See figure on previous page.)

Figure 3 Schematic diagram for genome regions surrounding spxB, ulaE and xfp locus in diverse lactobacilli. (A), spxB. (B), ulaE. (C), xfp. Gene syntenies were explored using the web service SyntTax [27]. TDF-derived protein sequences were used to query the selected genomes. Genes corresponding to query proteins are drawn in bold. A consistent color coding allows identification of orthologs and paralogs. Some gene names are indicated. Normalized BLAST scores are visualized. Reference organisms: L. rhamnosus GG, L. casei ATCC 334, L. buchneri CD034, L. plantarum WCFS1, L. helveticus R0052, L. delbrueckii subsp. bulgaricus ATCC 11842 and L. brevis ATCC 367. For ulaE, a conserved gene order was observed only in L. rhamnosus GG and L. casei ATCC 334.

The over-expression of $x f p$ mRNA levels in L. rhamnosus grown in $\mathrm{CB}$, as found in our study, seems to support this hypothesis. Moreover, our findings are in agreement with the proteomic data of Bove and colleagues [16], who observed an increase in expression level of ribose5-phosphate isomerase (Rpi) after L. rhamnosus growth in
CB compared to MRS. This enzyme acts in a step upstream of $\mathrm{xfp}$ in the pathway that leads from ribose 5-phosphate (R5P) to the production of acetate, catalyzing the conversion of R5P to ribulose 5-phosphate (Figure 5).

According to Pfam search, TDF 40-deduced 100 amino acid sequence contains a portion of the XFP C-terminal

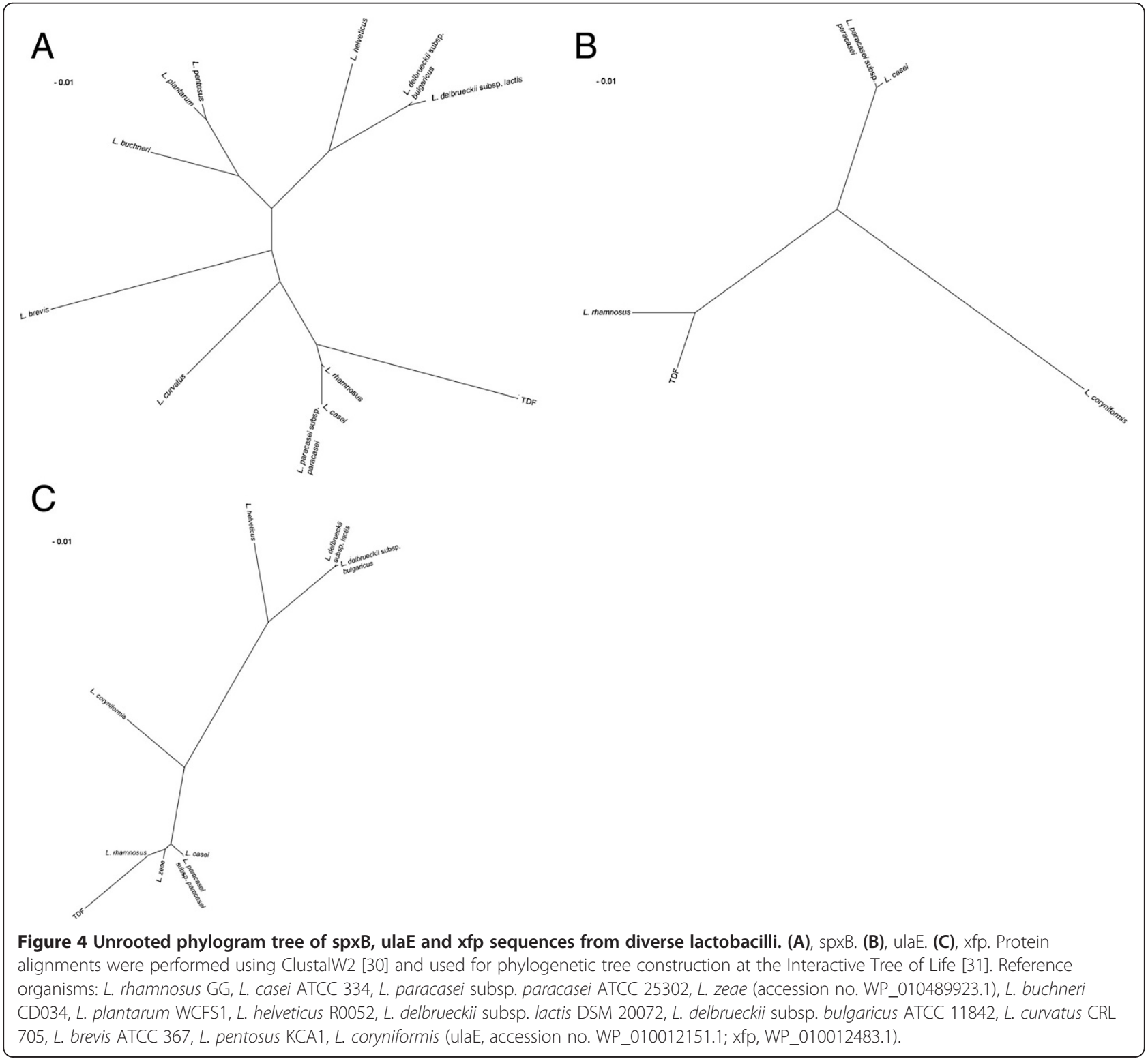




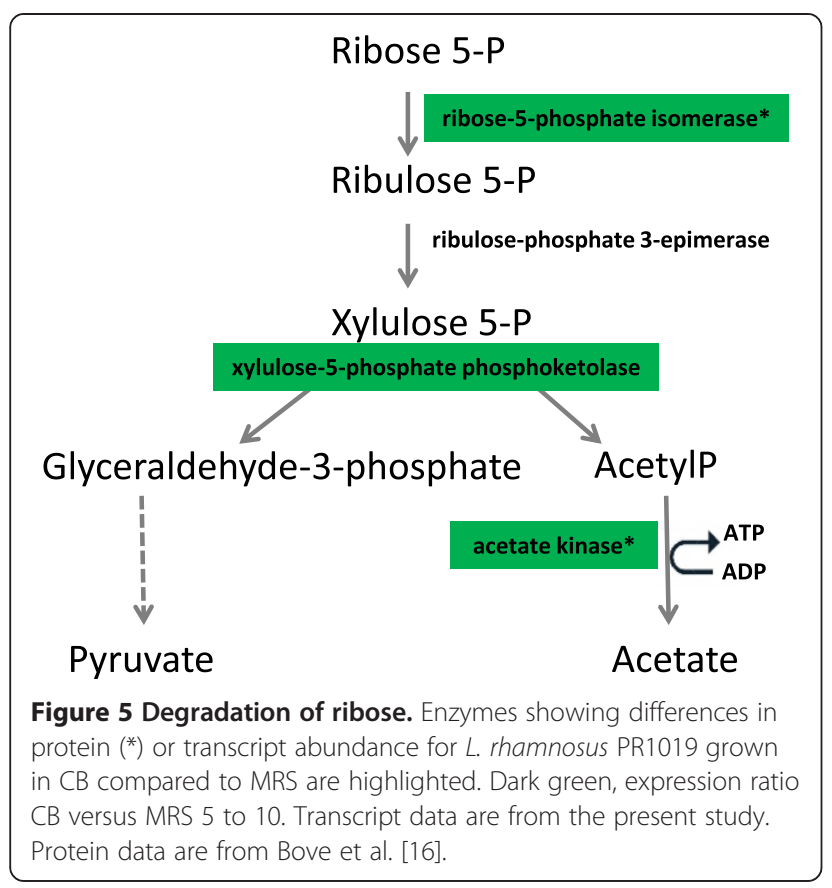

domain (pfam09363). The genetic organization and location of $x f p$ gene on L. rhamnosus GG and L. casei ATCC 334 chromosomes were shown to be highly similar (Figure $3 C$ ). In particular, $x f p$ genes are preceded by a divergently transcribed ORF, encoding a major facilitator superfamily transporter, and are followed by several genes predicted to encode components of $\mathrm{ABC}$ transporter and PTS systems for sugar uptake. According to PePPER, no high-scoring promoter consensus sequences were identified in the 5000-bp upstream region of $x f p$ gene in L. rhamnosus GG. A Rho-independent transcriptional terminator was predicted to occur $43 \mathrm{bp}$ downstream from the $x f p$ stop codon. Phylogram showed that $\mathrm{xfp}$ proteins from $L$. casei group made a separate cluster, close to the putative enzyme from $L$. coryniformis (Figure 4C). Analogously, different clusters were observed for the SLAB L. helveticus, L. delbrueckii subsp. lactis and L. delbrueckii subsp. bulgaricus. Additional file 1: Figure S1C displays a multiple sequence alignment of TDF 40 and putative phosphoketolases from several SLAB and NSLAB.

\section{Conclusions}

In this study, we applied a transcriptomic approach, based on CDNA-AFLP and qPCR, to investigate the physiological adaptation of $L$. rhamnosus to the cheese environment. L. rhamnosus is known to be one of the few NSLAB species able to survive and grow during long ripening of sseveral cheeses. In particular, the strain L. rhamnosus PR1019, isolated from 4-month-ripened PR cheese, has previously shown a great ability to growth in $\mathrm{CB}$ coupled with high levels of production of acetic acid. By comparing the gene expression profiles of $L$. rhamnosus PR1019 in CB respect to MRS, we identified among others as over-expressed in $\mathrm{CB}$, genes linked to the conversion of pyruvate to acetate as well as to the pathway of ribose degradation. Notably, the activation of POX pathway in L. rhamnosus has never been observed before.

Pyruvate is a intracellular metabolite that could be produced by different metabolism using the carbon source present in cheese and can be released in the cheese matrix with the starter lysis. Similarly the ribonucleosides release with starter lysis could be carriers of ribose that represents a fermentable carbohydrate in an environments such cheese where carbohydrates are lacking.

Both pyruvate degradation and ribose catabolism induce a metabolite flux toward acetate, coupled with ATP production via acetate kinase. Taking into account these consideration, and in agreement with previous findings [16] we assume that L. rhamnosus when growing in media poor in carbohydrates, such as $\mathrm{CB}$, arguably uses different metabolic pathways to produce energy. Notably, the transcriptomic approach employed in this study evidenced the over-expression in $\mathrm{CB}$ of enzymes other than those identified through proteomics by Bove et al. [16], acting at different steps or in different branches of the ribose and pyruvate utilization pathways. This discrepancy, probably owing to issues of technique sensitivity and resolution, highlighted the need to integrate transcriptomic and proteomic data in order to get a view as complete as possible of the L. rhamnosus metabolic adaptations during cheese ripening.

Since, to our knowledge, this is the first study that showed the activation of POX pathway in L. rhamnosus, further work will be directed to investigate more in depth the role of the pyruvate metabolism in the growth of this specie in cheese.

\section{Additional file}

Additional file 1: Figure S1. Multiple sequence alignment of spxB (A),
ulaE (B) and xfp (C) sequences from diverse lactobacilli. Conservation plots
and consensus sequences are shown at the bottom. Protein alignments were
performed and represented using CLC-Bio sequence viewer [32]. Reference
organisms: L. rhamnosus GG, L. casei ATCC 334, L. paracasei subsp. paracasei
ATCC 25302, L. zeae (accession no. WP_010489923.1), L. buchneri CD034,
L. plantarum WCFS1, L. helveticus R0052, L. delbrueckii subsp. lactis DSM
20072, L. delbrueckii subsp. bulgaricus ATCC 11842, L. curvatus CRL 705,
L. brevis ATCC 367, L. pentosus KCA1, L. coryniformis (ulaE, accession no.
WP_010012151.1; xfp, WP_010012483.1).

\section{Abbreviations}

LAB: Lactic acid bacteria; SLAB: Starter lactic acid bacteria; NSLAB: Non-starter lactic acid bacteria; L.: Lactobacillus; cDNA-AFLP: CDNA-amplified fragment length polymorphism; qPCR: Quantitative real-time reverse transcription-PCR; CB: Cheese broth; TDFs: Transcript-derived fragments; SpxB/POX: Pyruvate oxidase; UlaE: L-xylulose 5-phosphate 3-epimerase; Xfp: xylulose-5-phosphate phosphoketolase. 


\section{Competing interests}

The authors declare that they have no competing interests.

\section{Author's contributions}

CL: conceived the study, participated in its coordination and drafted the manuscript. ST: carried out genetic and bioinformatic analysis and helped to draft the manuscript. AM: carried out genetic analysis, participated in data collection and their interpretation. ES: carried out genetic analysis, participated in data collection and their interpretation. EN: critically revised the paper. PB: critically revised the paper. MG: conceived the study, supervised the research work and critically revised the paper. All authors read and approved the final manuscript.

\section{Acknowledgments}

The authors are grateful to Dr. Claudio Giorgio Bove for technical assistance.

\section{Author details}

${ }^{1}$ Department of Food Science, Parma University, Parco Area delle Scienze 48/A, 43124 Parma, Italy. ${ }^{2}$ Department of Pharmacy and Biotechnology, University of Bologna, Via Belmeloro 6, 40126 Bologna, Italy. ${ }^{3}$ Current address: Nutrition and Nutrigenomics Group, Department of Food Quality and Nutrition, Research and Innovation Centre, Fondazione Edmund Mach di San Michele all'Adige, Trento, Italy.

Received: 24 September 2013 Accepted: 3 February 2014 Published: 7 February 2014

\section{References}

1. Beresford TP, Fitzsimons NA, Brennan NL, Cogan T: Recent advances in cheese microbiology. Int Dairy J 2001, 11:259-274.

2. Sgarbi E, Lazzi C, lacopino L, Bottesini C, Lambertini F, Sforza S, Gatti M: Microbial origin of non proteolytic aminoacyl derivatives in long ripened cheeses. Food Microbiol 2013, 35:116-120.

3. Cogan TM, Beresford TP, Steele J, Broadbent J, Shah NP, Ustunol Z: Invited review: advances in starter cultures and cultured foods. J Dairy Sci 2007, 90:4005-4021.

4. Fox PF, McSweeney PLH: Cheese: an overview. In Cheese: Chemistry, Physics and Microbiology. General Aspects. 3rd edition. Edited by Fox PF, McSweeney PLH, Cogan TM, Guinee TP. London, UK: Elsevier; 2004:1-18.

5. Settanni L, Moschetti G: Non-starter lactic acid bacteria used to improve cheese quality and provide health benefits. Food Microbiol 2010, 27:691-697.

6. de Dea Lindner J, Bernini V, de Lorentiis A, Pecorari A, Neviani E, Gatti M: Parmigiano Reggiano cheese: evolution of cultivable and total lactic microflora and peptidase activities during manufacture and ripening. Dairy Sci Technol 2008, 88:511-523.

7. Santarelli M, Bottari B, Lazzi C, Neviani E, Gatti M: Survey on the community and dynamics of lactic acid bacteria in Grana Padano cheese. Syst Appl Microbiol 2013, 36:593-600.

8. Gatti M, de Dea Lindner J, de Lorentiis A, Bottari B, Santarelli M, Bernini V, Neviani E: Dynamics of whole and lysed bacterial cells during Parmigiano-Reggiano cheese production and ripening. Appl Environ Microbiol 2008, 74:6161-6167.

9. Neviani E, Bottari B, Lazzi C, Gatti M: New developments in the study of the microbiota of raw-milk, long-ripened cheeses by molecular methods: the case of Grana Padano and Parmigiano Reggiano. Front Microbiol 2013, 4:1-14.

10. Neviani E, de Dea Lindner E, Bernini V, Gatti M: Recovery and differentiation of long ripened cheese microflora through a new cheese-based cultural medium. Food Microbiol 2009, 26:240-245.

11. Bove CG, de Dea Lindner CG, Lazzi C, Gatti M, Neviani E: Evaluation of genetic polymorphism among Lactobacillus rhamnosus non-starter Parmigiano Reggiano cheese strains. Int J Food Microbiol 2011, 144:569-572.

12. Gatti M, Bottari B, Lazzi C, Neviani E, Mucchetti G: Invited review: Microbial evolution in raw-milk, long-ripened cheeses produced using undefined natural whey starters. J Dairy Sci 2014, 97:573-591.

13. Thomas TD: Cannibalism among bacteria found in cheese. N Z J Sci Technol Sect B 1987, 22:215-219.
14. Rapposch S, Eliskases-Lechner F, Ginzinger W: Growth of facultatively heterofermentative lactobacilli on starter cell suspensions. App/ Environ Microbiol 1999, 65:5597-5599.

15. Budinich MF, Perez-Díaz I, Cai H, Rankin SA, Broadbent JR, Steele JL: Growth of Lactobacillus paracasei ATCC 334 in a cheese model system: a biochemical approach. J Dairy Sci 2011, 94:5263-5277.

16. Bove CG, de Angelis M, Gatti M, Calasso M, Neviani E, Gobbetti M: Metabolic and proteomic adaptation of Lactobacillus rhamnosus strains during growth under cheese-like environmental conditions compared to de Man, Rogosa, and Sharpe medium. Proteomics 2012, 12:3206-3218.

17. de Man JC, Rogosa M, Elisabeth Sharpe M: A medium for the cultivation of lactobacilli. J App/ Microbiol 1960, 23:134-135.

18. Bove CG, Lazzi C, Bernini V, Bottari B, Neviani E, Gatti M: cDNA-amplified fragment length polymorphism to study the transcriptional responses of Lactobacillus rhamnosus growing in cheese-like medium. J Appl Microbiol 2011, 111:855-864.

19. Vuylsteke M, Peleman JD, van Eijk MJ: AFLP-based transcript profiling (CDNA-AFLP) for genome-wide expression analysis. Nat Protoc 2007, 2:1399-1413.

20. Ward LJ, Timmins MJ: Differentiation of Lactobacillus casei, Lactobacillus paracasei and Lactobacillus rhamnosus by polymerase chain reaction. Lett Appl Microbiol 1999, 29:90-92.

21. Blast. [http://blast.ncbi.n/m.nih.gov/Blast.cgi]

22. Turroni S, Bendazzoli C, Dipalo SC, Candela M, Vitali B, Gotti R, Brigidi P: Oxalate-degrading activity in Bifidobacterium animalis subsp. lactis: impact of acidic conditions on the transcriptional levels of the oxalyl coenzyme A $(\mathrm{COA})$ decarboxylase and formyl-CoA transferase genes. Appl Environ Microbiol 2010, 76:5609-5620.

23. Pfaffl MW: A new mathematical model for relative quantification in real-time RT-PCR. Nucleic Acids Res 2001, 29:e45.

24. Giraffa G, Lazzi C, Gatti M, Rossetti L, Mora D, Neviani E: Molecular typing of Lactobacillus delbrueckii of dairy origin by PCR-RFLP of protein-coding genes. Int J Food Microbiol 2003, 82:163-172.

25. Cluster of orthologous groups. [http://www.ncbi.nlm.nih.gov/COG/]

26. KEGG (Kyoto Encyclopedia of Genes and Genome). [http://www.genome. jp/kegg/pathway.html]

27. Oberto J: SyntTax: a web server linking synteny to prokaryotic taxonomy. BMC Bioinformatics 2013, 14:4.

28. de Jong A, Pietersma H, Cordes M, Kuipers OP, Kok J: PePPER: a webserver for prediction of prokaryote promoter elements and regulons. BMC Genomics 2012, 13:299.

29. Pfam motif analysis. [http://pfam.sanger.ac.uk/]

30. ClustalW2. [http://www.ebi.ac.uk/Tools/phylogeny/clustalw2_phylogeny/]

31. Tree of life. [http://itol.embl.de/index.shtml]

32. CLC-Bio sequence viewer. [http://www.clcbio.com/index.php?id=28]

33. Wang TT, Lee BH: Plasmids in Lactobacillus. Crit Rev Biotechnol 1997 17:227-272

34. Favier M, Bilhere E, Lonvaud-Funel A, Moine V, Lucas PM: Identification of pOENI-1 and related plasmids in Oenococcus oeni strains performing the malolactic fermentation in wine. PLoS One 2012, 7:49082.

35. Quatravaux S, Remize F, Bryckaert E, Colavizza D, Guzzo J: Examination of Lactobacillus plantarum lactate metabolism side effects in relation to the modulation of aeration parameters. J Appl Microbio/ 2006, 101:903-912.

36. Goffin P, Muscariello L, Lorquet F, Stukkens A, Prozzi D, Sacco M, Kleerebezem M, Hols P: Involvement of pyruvate oxidase activity and acetate production in the survival of Lactobacillus plantarum during the stationary phase of aerobic growth. Appl Environ Microbiol 2006, 72:7933-7940.

37. Lorquet F, Goffin P, Muscariello L, Baudry JB, Ladero V, Sacco M, Kleerebezem M, Hols P: Characterization and functional analysis of the poxB gene, which encodes pyruvate oxidase in Lactobacillus plantarum. J Bacteriol 2004, 186:3749-3759.

38. Murphy MG, Condon S: Correlation of oxygen utilization and hydrogen peroxide accumulation with oxygen induced enzymes in Lactobacillus plantarum cultures. Arch Microbiol 1984, 138:44-48.

39. Zotta T, Ricciardi A, Guidone A, Sacco M, Muscariello L, Mazzeo MF, Cacace G, Parente E: Inactivation of cсpA and aeration affect growth, metabolite production and stress tolerance in Lactobacillus plantarum WCFS1. Int J Food Microbiol 2012, 155:51-59.

40. Konings WN, Lolkema JS, Bolhuis H, van Veen HW, Poolman B, Driessen AJ: The role of transport processes in survival of lactic acid bacteria: energy 
transduction and multidrug resistance. Antonie Van Leeuwenhoek 1997, 7:117-128.

41. Brooijmans RJW, de Vos WM, Hugenholtz J: Lactobacillus plantarum WCFS1 electron transport chains. Appl Environ Microbiol 2009, 75:3580-3585.

42. Sgarbi E, Lazzi C, Tabanelli G, Gatti M, Neviani E, Gardini F: Nonstarter lactic acid bacteria volatilomes produced using cheese components. J Dairy Sci 2013, 96:4223-4234.

43. Liu SQ, Holland R, McJarrow P, Crow VL: Serine metabolism in Lactobacillus plantarum. Int J Food Microbiol 2003, 89:265-273.

44. Mortera P, Pudlik A, Magni C, Alarcon S, Lolkema JS: Ca2+-Citrate Uptake and Metabolism in Lactobacillus casei ATCC 334. Appl Environ Microbiol 2013, 79:4603-4612.

45. Shi R, Pineda M, Ajamian E, Cui Q, Matte A, Cygler M: Structure of L-xylulose-5Phosphate 3-epimerase (UlaE) from the anaerobic L-ascorbate utilization pathway of Escherichia coli: identification of a novel phosphate binding motif within a TIM barrel fold. J Bacteriol 2008, 190:8137-8144.

46. Yew WS, Gerlt JA: Utilization of L-ascorbate by Escherichia coli K-12: assignments of functions to products of the yjf-sga and yia-sgb operons. J Bacteriol 2002, 184:302-306.

47. Posthuma CC, Bader R, Engelmann R, Postma PW, Hengstenberg W, Pouwels PH: Expression of the xylulose 5-phosphate phosphoketolase gene, xpkA, from Lactobacillus pentosus MD363 is induced by sugars that are fermented via the phosphoketolase pathway and is repressed by glucose mediated by CcpA and the mannose phosphoenolpyruvate phosphotransferase system. Appl Environ Microbiol 2002, 68:831-837.

48. McLeod A, Snipen L, Naterstad K, Axelsson L: Global transcriptome response in Lactobacillus sakei during growth on ribose. BMC Microbiol 2011, 11:145.

49. Chaillou S, Champomier-Verges MC, Cornet M, Crutz-Le Coq AM, Dudez AM, Martin V, Beaufils S, Darbon-Rongere E, Bossy R, Loux V, Zagorec M: The complete genome sequence of the meat-borne lactic acid bacterium Lactobacillus sakei 23 K. Nat Biotechnol 2005, 23:1527-1533.

doi:10.1186/1471-2180-14-28

Cite this article as: Lazzi et al:: Transcriptomic clues to understand the growth of Lactobacillus rhamnosus in cheese. BMC Microbiology 2014 14:28.

\section{Submit your next manuscript to BioMed Central and take full advantage of:}

- Convenient online submission

- Thorough peer review

- No space constraints or color figure charges

- Immediate publication on acceptance

- Inclusion in PubMed, CAS, Scopus and Google Scholar

- Research which is freely available for redistribution 